\title{
Atomic resolution mapping using quantitative high-angle annular dark field scanning transmission electron microscopy
}

\author{
S. Van Aert*, J. Verbeeck*, S. Bals*, R. Erni**, D. Van Dyck*, and G. Van Tendeloo* \\ *Electron Microscopy for Materials Science (EMAT), University of Antwerp, Groenenborgerlaan \\ 171, 2020 Antwerp, Belgium \\ **National Center for Electron Microscopy, Ernest Orlando Lawrence Berkeley, National \\ Laboratory, 1 Cyclotron Road, MS 72R0150, Berkeley, CA 94720, USA
}

It is generally known that HAADF STEM images show Z-contrast. One of the advantages is therefore the possibility to visually distinguish between chemically different atomic column types. However, if the difference in atomic number of distinct atomic column types is small or if the signalto-noise ratio is poor, direct interpretation of HAADF STEM images is inadequate. Moreover, if the tails of the electron probe have contributions on neighboring atomic columns, intensity transfer from one atomic column to neighboring atomic columns may occur. Such effects also hamper the direct interpretation of the images, especially when studying interfaces.

In order to extract local chemical information with good accuracy and precision, a new quantitative HAADF STEM method has been developed [1]. The method is based on a quantification of the total intensity of the scattered electrons for the individual atomic columns using statistical parameter estimation theory. In order to apply this theory, a model is required describing the image contrast of the HAADF STEM images. Therefore, a simple, effective incoherent model has been assumed: a parameterized object function, which is peaked at the atomic column positions, convoluted with the probe intensity profile. Next, the scattered intensities, corresponding to the volumes under the peaks of the object function, are estimated by fitting this model to experimental HAADF STEM images. These estimates can then be used as a performance measure to distinguish between different atomic column types and to identify the nature of unknown columns using statistical hypothesis testing. The reliability of the method is supported by means of simulated HAADF STEM images as well as a combination of experimental images and electron energy-loss spectra.

It has experimentally been shown that statistically meaningful information on the composition of individual columns can be obtained even if the difference in averaged atomic number $\mathrm{Z}$ is only 3. Figure 1a shows an enlarged area from an experimental HAADF STEM image of a $\mathrm{La}_{0.7} \mathrm{Sr}_{0.3} \mathrm{MnO}_{3}$ $\mathrm{SrTiO}_{3}$ multilayer structure close to the $\mathrm{SrTiO}_{3}$ substrate using an FEI TITAN 80-300 microscope operated at $300 \mathrm{kV}$. Note that no visual conclusions could be drawn concerning the sequence of the atomic planes at the interfaces. The refined model is shown in figure 1b. Figure 1c shows the experimental observations together with an overlay indicating the estimated positions of the columns together with their atomic column types. The composition of the columns away from the interfaces is assumed to be known whereas the composition of the columns in the planes close to the interface (indicated by the symbol ' $\mathrm{X}$ ') is unknown. Histograms of the estimated peak volumes of the known columns are presented in figure 2 and show the statistical nature of the result. The colored vertical bands correspond to $90 \%$ tolerance intervals. It is important to note that these tolerance intervals are not overlapping meaning that columns, even for which the difference in averaged atomic number is only 3 ( $\mathrm{TiO}$ and $\mathrm{MnO}$ ), can clearly be distinguished. Next, in order to identify an unknown column, its estimated peak volume is compared with the colored confidence intervals. Its column type is equal to the column type corresponding to the tolerance interval containing the estimated peak 
volume of the unknown column. A column whose estimated peak volume falls in between the tolerance intervals corresponding to $\mathrm{Z}_{\mathrm{i}}$ and $\mathrm{Z}_{\mathrm{j}}$ will have an averaged atomic number in between $\mathrm{Z}_{\mathrm{i}}$ and $Z_{j}$. For this multilayer structure, such effects are the result of intermixing or diffusion of different types of atoms [2]. Single-colored dots are used to indicate columns whose estimated peak volume falls inside a tolerance interval whereas pie charts are used otherwise. The sizes of the two segments are an indication of the relative position of the estimated peak volume with respect to the nearest tolerance intervals. The results of this quantification are shown on the right-hand side of figure 1c. The lower planes of the quantified areas consist of purely $\mathrm{TiO}$ and $\mathrm{MnO}$ columns, respectively. However, intermixing of $\mathrm{La}$ and $\mathrm{Sr}$ and of $\mathrm{Ti}$ and $\mathrm{Mn}$ is observed in the upper two planes. The degree of intermixing is more important for the interface on top of the STO layer than for the interface at the bottom of the STO layer. These results have been confirmed by an experimental EELS line scan proving that the method works well in practice [3].

[1] S. Van Aert et al., Ultramicroscopy (2009) submitted.

[2] D. A. Muller et al., Science 319 (2008) 1073.

[3] The authors acknowledge financial support from the Fund for Scientific Research - Flanders (FWO). Financial support from the European Union for the Framework 6 program under a contract for an Integrated Infrastructure Initiative (Reference 026019 ESTEEM) is acknowledged.
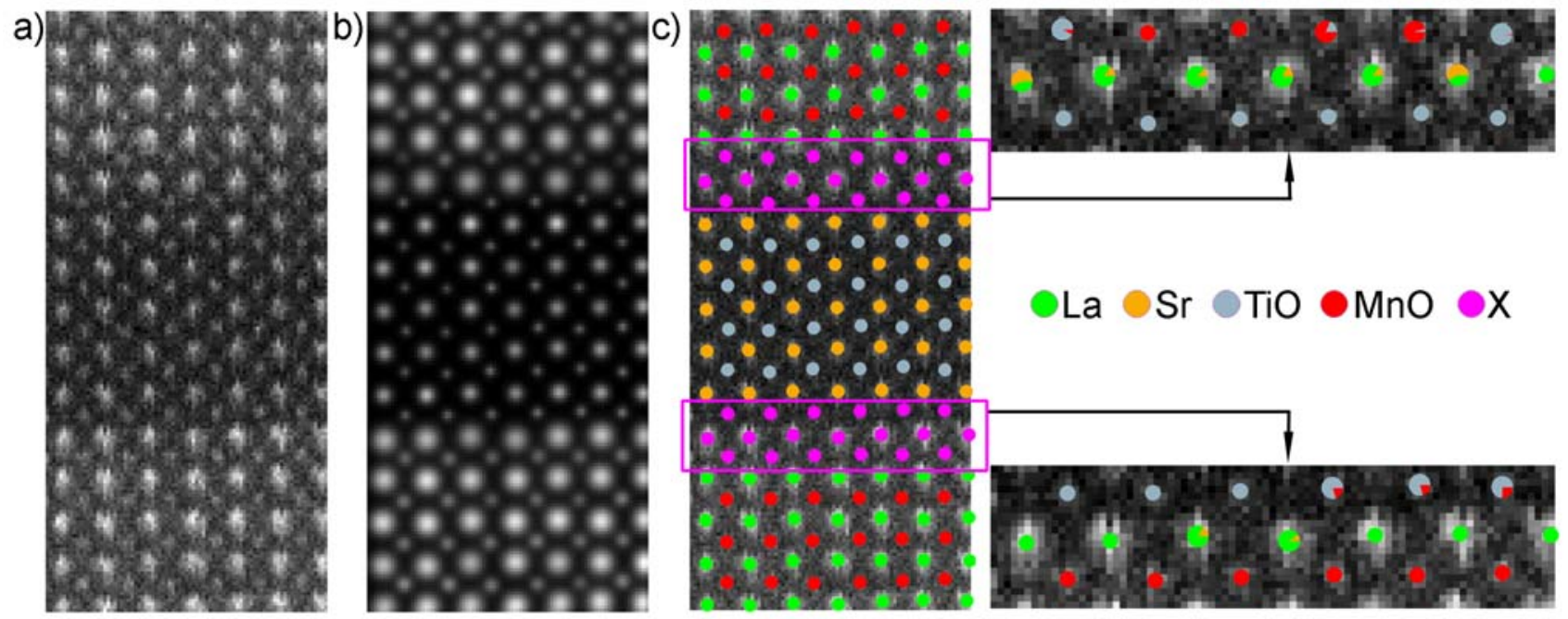

FIG. 1. (Color online) (a). Area from an experimental HAADF STEM image. (b) Refined model. (c) Overlay indicating the estimated positions of the columns together with their atomic column types.

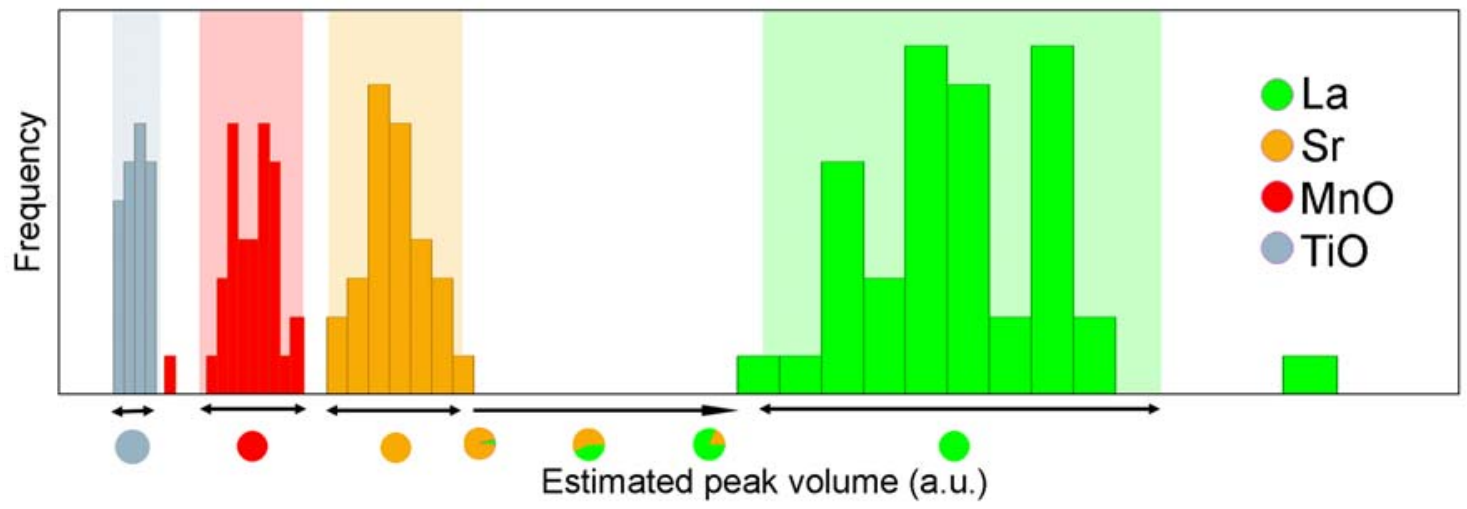

FIG. 2. (Color online) Histograms of the estimated peak volumes of the known columns. 